\title{
The Yersinia enterocolitica type three secretion chaperone SycO is integrated into the Yop regulatory network and binds to the Yop secretion protein YscMI
}

\author{
Svea Dittmann ${ }^{1}$, Annika Schmid ${ }^{1}$, Susanna Richter ${ }^{1}$, Konrad Trülzsch ${ }^{1}$, \\ Jürgen Heesemann ${ }^{1}$ and Gottfried Wilharm*1,2
}

Address: ${ }^{1}$ Max von Pettenkofer-Institut, Lehrstuhl für Bakteriologie, Ludwig-Maximilians-Universität München, Pettenkoferstr. $9 \mathrm{a}$, D-80336 München, Germany and 2Robert Koch-Institut, Bereich Wernigerode, Burgstr. 37, D-38855 Wernigerode, Germany

Email: Svea Dittmann - dittmann@mvp.uni-muenchen.de; Annika Schmid - schmid_a@mvp.uni-muenchen.de; Susanna Richter - richter_s@mvp.uni-muenchen.de; Konrad Trülzsch - truelzsch@mvp.uni-muenchen.de; Jürgen Heesemann - heesemann@mvp.uni-muenchen.de; Gottfried Wilharm* - wilharmg@rki.de

* Corresponding author

Published: 5 July 2007

BMC Microbiology 2007, 7:67 doi:10.1 186/147/-2180-7-67
Received: 29 November 2006

Accepted: 5 July 2007

This article is available from: http://www.biomedcentral.com/I47I-2/80/7/67

(c) 2007 Dittmann et al; licensee BioMed Central Ltd.

This is an Open Access article distributed under the terms of the Creative Commons Attribution License (http://creativecommons.org/licenses/by/2.0), which permits unrestricted use, distribution, and reproduction in any medium, provided the original work is properly cited.

\begin{abstract}
Background: Pathogenic yersiniae (Y. pestis, Y. pseudotuberculosis, Y. enterocolitica) share a virulence plasmid encoding a type three secretion system (T3SS). This T3SS comprises more than 40 constituents. Among these are the transport substrates called Yops (Yersinia outer proteins), the specific Yop chaperones (Sycs), and the Ysc (Yop secretion) proteins which form the transport machinery. The effectors YopO and YopP are encoded on an operon together with SycO, the chaperone of YopO. The characterization of SycO is the focus of this study.

Results: We have established the large-scale production of recombinant SycO in its outright form. We confirm that $Y$. enterocolitica SycO forms homodimers which is typical for Syc chaperones. SycO overproduction in $Y$. enterocolitica decreases secretion of Yops into the culture supernatant suggesting a regulatory role of SycO in type III secretion. We demonstrate that in vitro SycO interacts with YscMI, a negative regulator of Yop expression in $Y$. enterocolitica. However, the SycO overproduction phenotype was not mediated by YscMI, YscM2, YopO or YopP as revealed by analysis of isogenic deletion mutants.

Conclusion: We present evidence that SycO is integrated into the regulatory network of the Yersinia T3SS. Our picture of the Yersinia T3SS interactome is supplemented by identification of the $\mathrm{SycO} / \mathrm{YscMI}$ interaction. Further, our results suggest that at least one additional interaction partner of SycO has to be identified.
\end{abstract}

\section{Background}

An ever increasing number of Gram-negative bacteria is known to utilize a type three secretion system (T3SS), mostly pathogens that manipulate their host cells by injecting effector proteins [1]. One of the characteristics of
T3SSs is the existence of specific energy-independent chaperones which assist several of the transport substrates. Chaperones assisting the effectors (class I chaperones) are distinguished from those assisting the pore forming translocators (class II chaperones) [2]. The mode 
of interaction between class I chaperones and their respective substrates is well-investigated, mainly due to crystal structures of several chaperone/substrate complexes [3-5]. Typically, a region of 50-80 amino acids following the putative $\mathrm{N}$-terminal secretion signal is wrapped around a chaperone dimer thereby preventing tertiary structure formation of this substrate region. The rest of the substrate, however, is folded [4-8]. The chaperone/substrate complexes are recognized by a T3SS-specific ATPase [9], which is able to displace the chaperone from its substrate and further to act as an unfoldase unraveling the substrate [10].

Despite these recent discoveries, the substrate recognition process has remained enigmatic $[11,12]$. It has been suggested that the chaperone/substrate complexes may constitute a three-dimensional secretion signal [4]. This hypothesis is supported by the intriguing fact that the structures of all classI chaperones share a common fold even across the genus barrier and despite low sequence similarity.

The T3SS of pathogenic Yersinia spp. is among the most intensively studied T3SSs with the structures of the homodimeric chaperones SycE [13-15], SycH [16], SycT $[17,18]$, and the atypical SycN/YscB heterodimer [5] being solved. According to phylogenetic analyses and experimental results, the Yersinia T3SS holds at least one additional classI chaperone, SycO $[19,20]$.

Here, we focus on characterization of $Y$. enterocolitica SycO, encoded by a gene termed orf 155 until recently. In $Y$. enterocolitica, sycO is organized in an operon together with yopO ( $y p k A$ in Y. pseudotuberculosis and Y. pestis) and yopP ( yopJ in Y. pseudotuberculosis and Y. pestis). Within this operon, sycO is located upstream of the yopO gene. A nonpolar deletion mutant of orf1, the sycO orthologue in $Y$. pseudotuberculosis, secreted YpkA and YopJ in amounts comparable to the wildtype and was fully virulent. Further, orf1 was not found to be involved in temperature and calcium-dependent control of Yop expression and secretion [21]. Because of these results and the use of the infrequent "GTG" start codon of orf1, Galyov et al. [21] proposed that orf1 might not be translated. Later, the corresponding orf7 of $Y$. pestis was discussed as a potential Syc-like chaperone due to the typical size $(15.7 \mathrm{kDa})$, isoelectric point (pI of 4.39), and amphipathic character of the putative translation product [22]. However, in Y. enterocolitica the induction of apoptosis by YopP in macrophages was shown to be independent of orf155, and it was found that secretion of YopO into the culture supernatant was apparently not restricted in the absence of orf155 [23]. Only recently, a chaperone function of the orf 155 product on the effector YopO was reported. Consequently, the gene product of orf155 was termed SycO and the gene was renamed sycO [20].

Here, we characterize recombinantly produced $\mathrm{SycO}$ as a homodimer. We demonstrate the potential of SycO to influence Yop expression and secretion, and identify a novel interaction partner of SycO.

\section{Results}

\section{Recombinant production of SycO}

To examine SycO in vitro, we recombinantly produced SycO in E. coli and purified it to near homogeneity. For this purpose, we followed a protocol previously established for production of SycH and SycT to generate the proteins in their outright form without an affinity tag $[7,18]$. The sycO sequence of $Y$. enterocolitica strain WA314 [24] was amplified by PCR, thereby changing the start codon from "GTG" to "ATG" to ensure maximal expression. The fragment was cloned into vector pWS [18]. Expression was performed in E. coli BL21 (DE3) pLysS. SycO was produced in abundance and its solubility was optimized by expression at $27^{\circ} \mathrm{C}$ overnight. A purification protocol was established combining ammonium sulfate precipitation followed by MonoQ-based anion exchange chromatography and a final gel filtration step. The yield was approximately $15 \mathrm{mg}$ of purified protein per liter of culture with a homogeneity of about $95 \%$.

\section{Recombinant SycO forms homodimers}

Typically, T3SS effector chaperones form homodimers. In order to test SycO for oligomerization, we applied analytical gel filtration and chemical cross-linking. Fig. 1A depicts the SDS-PAGE analysis of a Superdex75 gel filtration of a mixture of recombinant SycO $(15.9 \mathrm{kDa})$ and the monomeric marker protein carbonic anhydrase $(29 \mathrm{kDa})$. The Coomassie-stained gel of consecutive fractions shows that $\mathrm{SycO}$ elutes before the $29 \mathrm{kDa}$ marker protein. Calibration of the column revealed an apparent molecular mass of approximately $35 \mathrm{kDa}$ for SycO, which is well in accordance with dimer formation. To confirm this, we performed a chemical cross-linking experiment as shown in Fig.1B. The cross-linking reagent DSP generated heatstable coupling products corresponding to SycO dimers as resolved by SDS-PAGE. Note that the mobility of SycO in SDS-PAGE is higher than that of typical marker proteins due to its low isoelectric point of 4.3.

\section{Overproduction of SycO represses Yop secretion in $\mathrm{Y}$. enterocolitica}

It was described that overproduction of the regulatory T3SS chaperone SycH in Yersinia influences regulation of Yop secretion and expression $[25,26]$. In order to test SycO for possible regulatory functions, we overproduced SycO in Y. enterocolitica WA-314 and in the isogenic $\Delta y o p O$ and $\Delta y o p P$ mutant strains [27]. In all three strains, the 

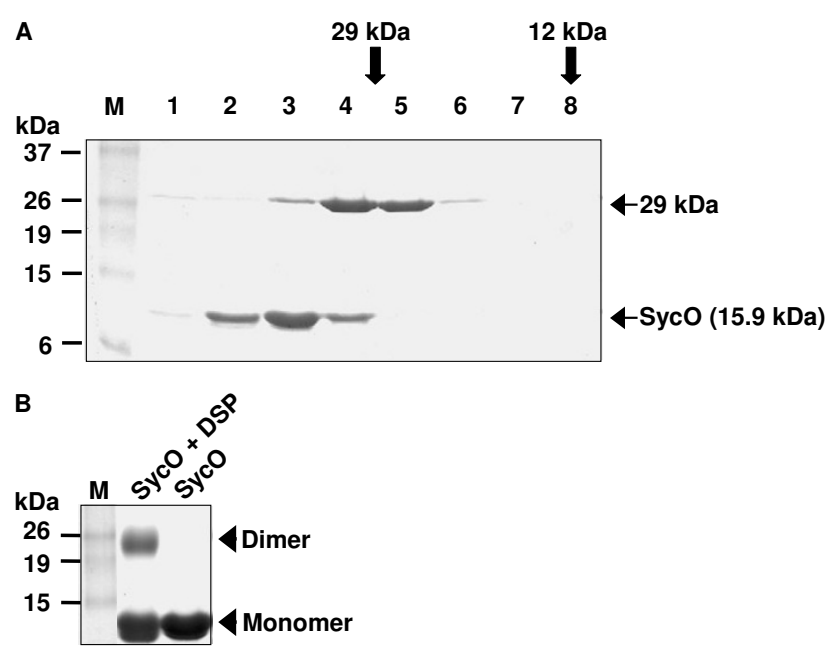

Figure I

Recombinant SycO forms homodimers. (A) Analytical gel filtration shows that SycO elutes from a Superdex 75 column prior to the $29 \mathrm{kDa}$ marker protein, carbonic anhydrase. Consecutive fractions were analyzed by SDS-PAGE (Coomassie-stained); the column was calibrated as indicated above the gel. (B) Chemical cross-linking of recombinant SycO using DSP as cross-linking reagent was analyzed by Coomassie-stained SDS-PAGE.

overproduction of SycO reduced the level of secreted Yops significantly (Fig. 2A). An IPTG titration series is shown in Fig. 2B illustrating the correlation between an increase of SycO production and a decrease of Yop secretion. As an exception, the level of secreted YopO remained stable upon SycO overproduction in the parental strain and in the $\triangle$ үорP mutant strain as revealed by Western blotting (Fig. 2A).

Strikingly, analysis of the pellet fractions revealed a decrease of all Yops including YopO within the cells overproducing SycO (Fig. 2C). It was recently demonstrated, that secretion of YopO into the culture supernatant does not strictly rely on SycO $[20,23]$. Thus, excess of SycO could increase the secretion efficiency of YopO. The finding that the amount of secreted YopO was constant despite of reduced intracellular levels suggests that YopO secretion efficiency was increased by excess of SycO.

On closer inspection of YopP secretion, it became evident that deletion of $y \circ p O$ generated a polar effect resulting in reduced YopP secretion (Fig. 2A). Interestingly, SycO overproduction did not cause as pronounced a reduction of YopP secretion in the yopO mutant as in the parental strain. This observation might point to an interrelation between $\mathrm{YopO} / \mathrm{SycO}$ and YopP and hence to an explanation for the operon structure.
Taken together, these results suggest that SycO overproduction interferes with the T3SS regulatory network. Strikingly, this effect is neither mediated by an interaction of SycO with its effector YopO nor by an interaction with YopP which is also encoded within the same operon.

To test a possible unspecific effect of $\mathrm{SycO}$ overproduction, we searched for a protein with similar functions and properties but unrelated to the T3SS to overproduce it as a control. We chose SecB, the chaperone of the Sec transport pathway, which exhibits similar properties compared to SycO (SecB: $17.3 \mathrm{kDa}$, pI 3.91; SycO: $15.9 \mathrm{kDa}, \mathrm{pI}$ 4.32). Overproduction of SecB in the same strains did not cause any significant effect on secretion and expression of Yops (Fig. 3), suggesting a specific effect of SycO towards the T3SS.

As a further control of specificity, we analyzed the influence of SycO overproduction on the Sec-pathway by monitoring beta-lactamase-mediated resistance to carbenicillin. We found no influence of SycO overproduction on the level of carbenicillin resistance even at concentrations above $1 \mathrm{mg} / \mathrm{ml}$ of carbenicillin (data not shown).

Finally, we compared the effect of SycO overproduction to that of SycH overproduction (Fig. 4). Even though the overproduction of $\mathrm{SycH}$ was very massive compared to SycO (Fig. 4B) no similar effect on secretion of Yops was observed (Fig. 4A). In conclusion, these findings suggest that the SycO overexpression phenotype is due to a specific interaction of $\mathrm{SycO}$ with some component of the T3SS or one involved in the T3SS regulatory network.

\section{Differential effect of SycO overproduction on accumulation of Yops in the Yersinia cytosol in the presence of calcium ions}

We further analyzed the effect of SycO overproduction in the presence of $5 \mathrm{mM} \mathrm{CaCl}_{2}$ at $37^{\circ} \mathrm{C}$ to detect a possible calcium-blind phenotype. We observed that none of the strains secreted Yops in the presence of calcium (data not shown). Interestingly, SycO overproduction increased the cytosolic YopO levels (Fig. 5). An excess of SycO possibly stabilizes YopO and thereby increases its half-life when no secretion takes place. In comparison, YopH levels were not significantly affected upon overproduction of $\mathrm{SycO}$ (Fig. 5). Consequently, SycO overproduction interferes with Yop expression only under conditions of Yop secretion.

\section{SycO interacts with the T3SS regulatory protein YscMI}

Several Yersinia T3SS chaperones like SycD, SycE, and $\mathrm{SycH}$ are known to be involved in regulation of Yop expression $[26,28,29]$. Interestingly, these chaperones interact with the negative regulators of Yop expression, YscM1 (orthologous to LcrQ of Y. pestis and Y. pseudotuber- 

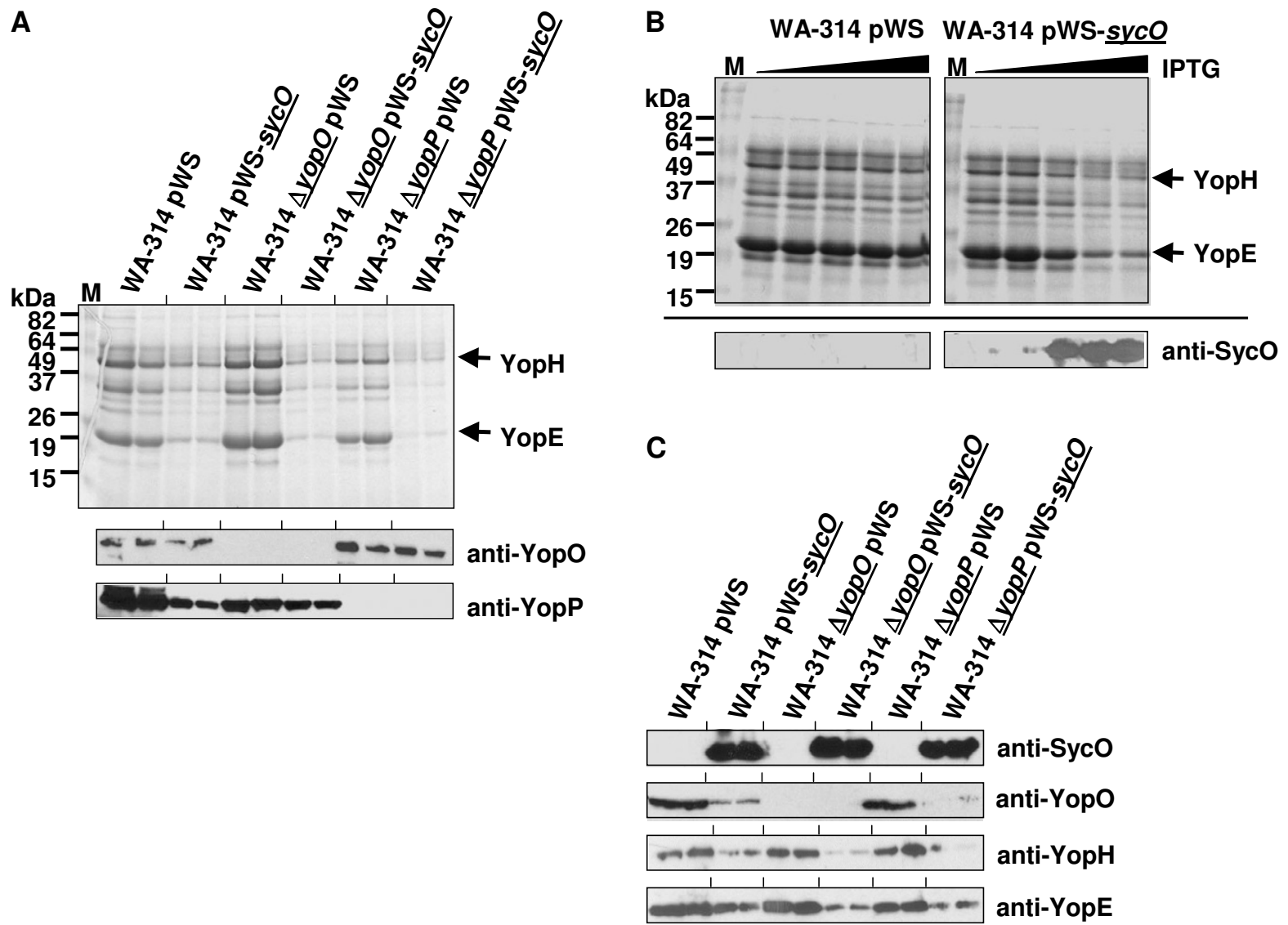

\section{Figure 2}

Overproduction of SycO represses secretion and expression of Yops. Y. enterocolitica parental strain WA-3I4 and the isogenic deletion mutants $\triangle y o p O$ and $\triangle y o p P$ were transformed with plasmids pWS-sycO and pWS, respectively. After culturing at $37^{\circ} \mathrm{C}$ in the presence of $0.2 \mathrm{mM} \mathrm{CaCl}$ for $2 \mathrm{~h}$, Yop secretion was induced with EGTA. Simultaneously, the cultures were supplemented with I mM IPTG and grown for another $2 \mathrm{~h}$. (A) TCA-precipitated culture supernatants were analyzed by Coomassiestaining of an SDS gel (upper panel), and by immunoblotting of YopO and YopP (lower panels). Two independent cultures of each strain are represented. Calibration of gel loads was obtained by measurement of the optical density of the cultures. (B) Concentration-dependency of the SycO overproduction phenotype. Yop secretion was induced in Y. enterocolitica WA-3I4 harboring plasmids pWS-sycO and pWS, respectively, and simultaneously SycO overproduction was induced by addition of IPTG in increasing amounts (indicated by the filled triangles). The IPTG concentrations were 0, 0.00I, 0.0I, 0.I, and I mM, respectively. The upper panel shows a Coomassie-stained SDS gel after separation of TCA-precipitated supernatants. The lower panel shows an immunoblot of bacterial pellets to detect the expression of SycO. (C) Cell lysates corresponding to the secretion analysis presented in $(\mathrm{A})$ were analyzed by immunoblotting of SycO, YopO, YopH, and YopE.

culosis) and YscM2, respectively [25,30,31]. YscM2 is only present in Y. enterocolitica and paralogous to YscM1, sharing 57\% identical residues [32]. Since our results suggest a regulatory function of SycO, we speculated that SycO might also interact with YscM1 and YscM2. To check these interactions, we performed native gel electrophoresis as shown in Fig. 6. A representative Coomassie-stained native gel (Fig. 6, panel A) shows that loading a mixture of the recombinant proteins YscM1 and SycO results in appearance of an additional band (lane 2). This band does not appear when loading either YscM1 (lane 1) or SycO (lane 3) alone. Immunoblots developed with antisera raised against SycO (panel B) and YscM1 (panel C) revealed that this band represents a complex composed of YscM1 and SycO. In contrast, no interaction between YscM2 and SycO was found (lane 4). Note that due to the high isoelectric point of YscM2 (pI 9.79) this protein does not enter the native gel with a $\mathrm{pH}$ of 8 [31]. 

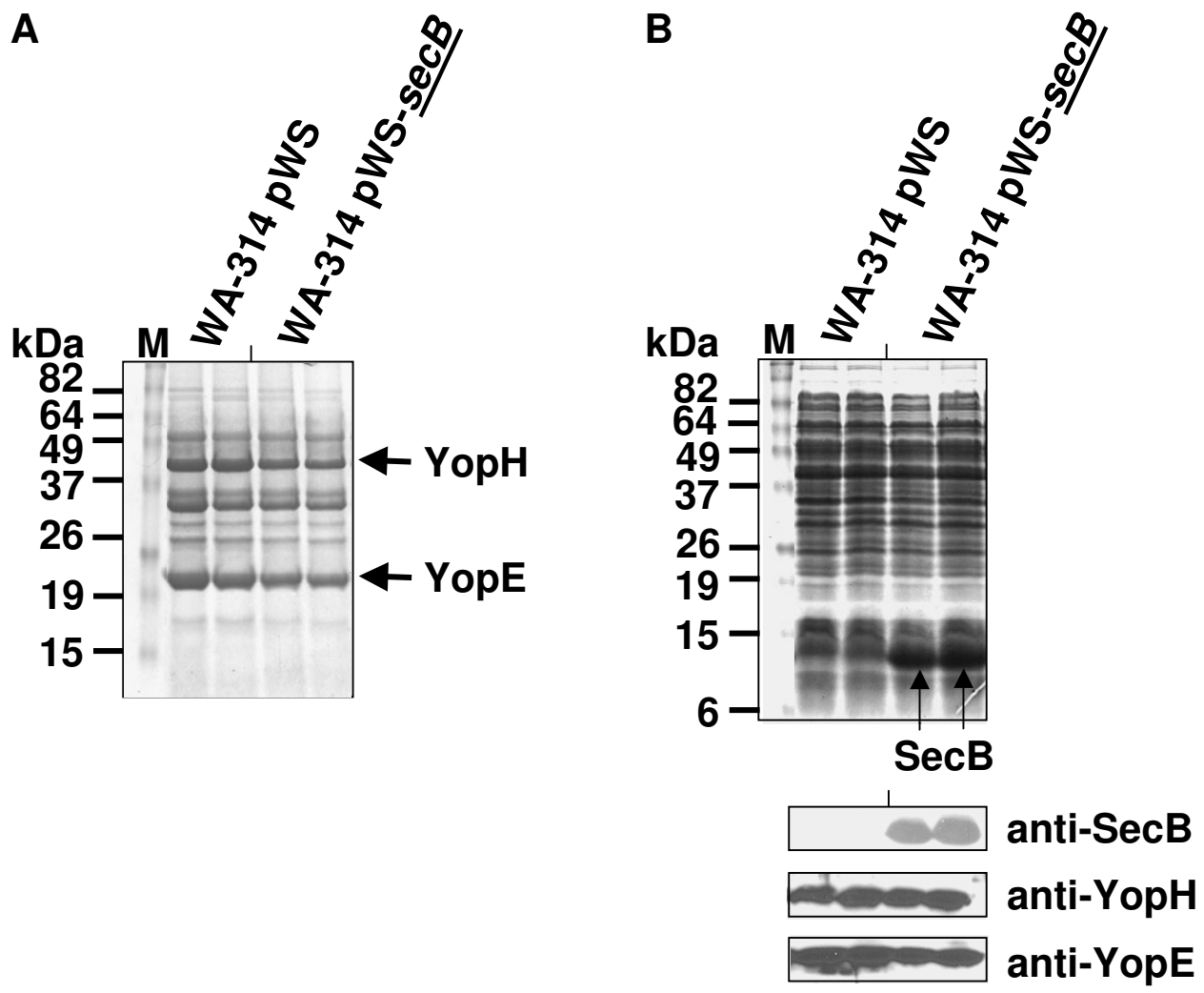

\section{Figure 3}

Overproduction of $\mathrm{SecB}$ does not cause significant effects on secretion and expression of Yops. Analogous to the experimental setup described in legend to Fig. 2, SecB was overproduced. (A) Coomassie-stained SDS-PAGE after loading of TCA-precipitated fractions of the culture supernatant. (B) Analysis of the corresponding cell lysates; Coomassie-stained gel (upper panel) and corresponding immunoblots to detect $\mathrm{SecB}, \mathrm{YopH}$, and YopE (lower panels).

Cross-linking experiments were performed to verify the YscM1/SycO interaction. However, the data did not help to interpret the results from native gel electrophoresis since YscM1 and SycO are very similar in size and both form homodimers ([33], Fig. 1). As a consequence, the major cross-linking products could not be unambiguously assigned to heterocomplexes (data not shown).

\section{Overproduction of SycO in the yscMI and yscM2 deletion mutants}

We speculated that the effects of SycO overproduction described above could be mediated by the regulatory proteins YscM1 and YscM2. To find out whether such a connection exists, we generated deletion mutants of $y s c \mathrm{M1}$ and $y s c M 2$, respectively, to use them as background for SycO overproduction. Deletions of $y s c M 1$ and $y s c M 2$ were generated by the Red recombinase based method of Datsenko and Wanner [34] that was adapted to Y. enterocolitica [35]. In accordance with Stainier et al. [32], we found that neither the $\Delta y s c M 1$ nor the $\Delta y s c M 2$ mutant exhibited a phenotype with respect to Yop expression and secretion (Fig. 7). Overall, overproduction of SycO in both mutant strains caused a decrease of Yop secretion (Fig. 7A) and expression (Fig. 7B) similar to what was observed for the parental strain. However, two reproducible phenomena related to the $y s c M 1$ deletion strain could be observed. First, SycO overproduction caused a decrease of YopO secretion (Fig. 7A). In the presence of extracellular calcium, the increase of YopO levels was less pronounced in the $\Delta y s c M 1$ and also in the $\Delta y s c M 2$ strain compared to the parental strain (Fig. 8). Second, decrease of YopP secretion as a consequence of SycO overproduction was more pronounced in the $\Delta y s c M 1$ strain compared to any other strain (Fig. 7A and Fig. 2A for comparison).

In summary, these results show that neither YscM1 nor YscM2 is required to establish the SycO-mediated shutdown of Yop expression and secretion. However, the yscM1 deletion mutant exhibits a phenotype suggesting 

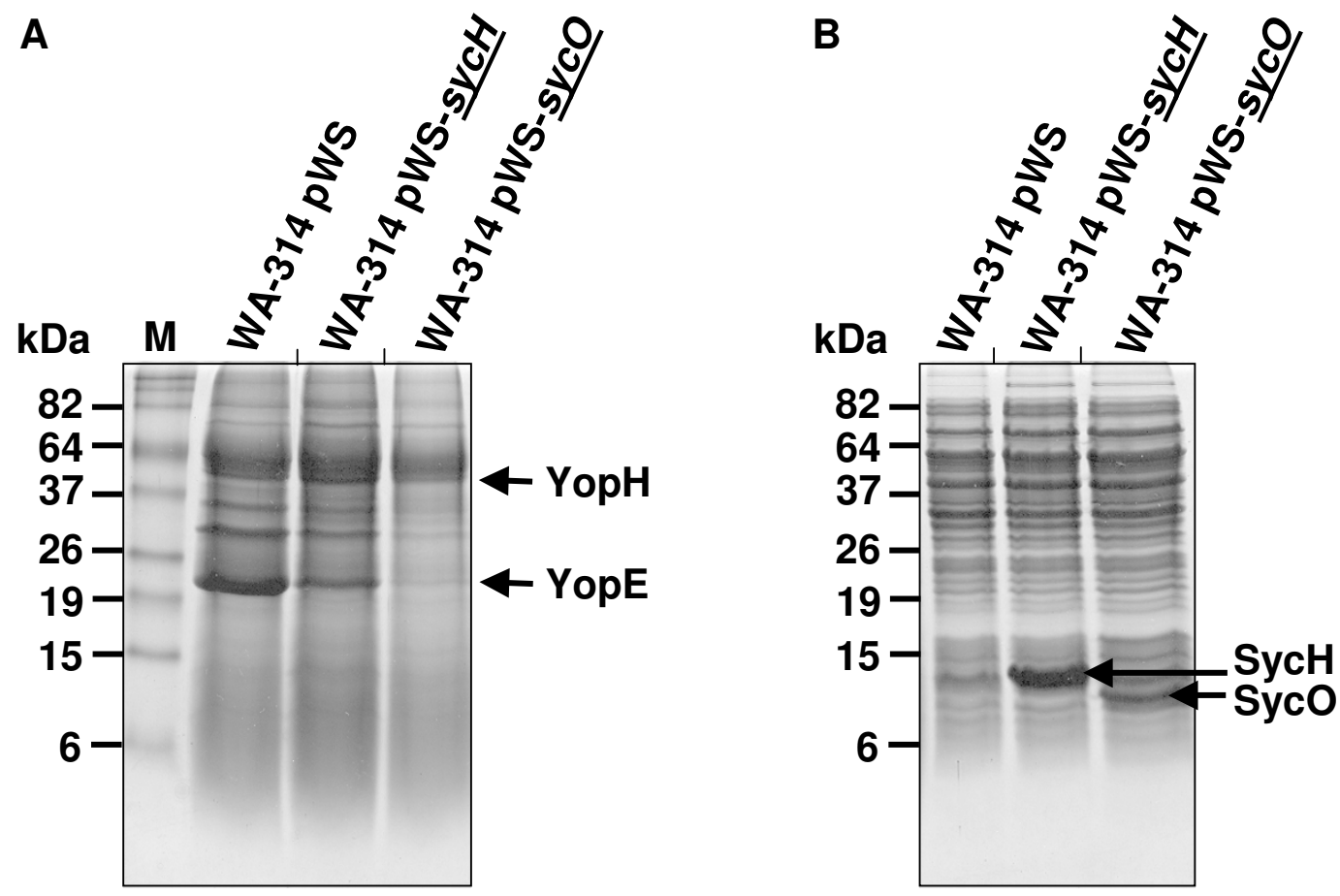

\section{Figure 4}

The SycH overproduction phenotype differs significantly from that of SycO. Analogous to the experimental setup described in legend to Fig. 2, SycH and SycO were overproduced in yersiniae. (A) Coomassie-stained SDS-PAGE after loading of TCA-precipitated fractions of the culture supernatant. (B) Coomassie-stained gel of the corresponding cell lysates.

that SycO and YscM1 act together in the regulation of YopO and YopP.

\section{Discussion}

In this study, we demonstrate that $Y$. enterocolitica SycO behaves as a homodimer in solution. This is in full agreement with a recent study from Letzelter et al. [20] who also applied gel filtration analysis and cross-linking techniques with the same outcome. Homodimer formation is typical for T3SS chaperones of effectors, and SycO dimerization is thus plausible in the light of the recent discovery that SycO serves as a chaperone for YopO [20]. Now, that we have established a protocol for the large-scale production of native SycO we aim at generating high-resolution structural data to characterize $\mathrm{SycO}$ on the molecular level.

We have further presented evidence that the functions of SycO are not restricted to chaperoning YopO. We could show that overproduction of $\mathrm{SycO}$ causes an apparent repression of Yop secretion and expression. Two principle explanations for this phenomenon seem plausible. On the one hand, SycO could interfere with the regulatory network involved in Yop expression, thereby causing down-regulation of Yop expression and consequently reducing the amount of secreted Yops. On the other hand, SycO could interfere with some component of the T3SS transport machinery. In particular, T3SS chaperones are capable of interacting with the specific ATPase of the transport machinery [9] irrespective of effector-binding to the chaperones. Thus, abundant SycO could compete with chaperone/Yop complexes and thereby reduce the efficiency of Yop secretion. Since a shutdown of secretion typically results in feedback inhibition of Yop expression, this scenario could explain the phenotype equally well. This latter hypothesis is challenged by the observation that overproduction of $\mathrm{SycH}$ in $Y$. pestis causes a slight increase of Yop expression and secretion as well as a relaxation of the Calcium-dependent control of Yop secretion [26]. Similarly, moderate overproduction of SycH induces Yop production in Y. enterocolitica and Y. pseudotuberculosis [25]. Even the dramatically elevated SycH levels that we applied could not provoke an effect comparable to that of SycO. Therefore, an interference of SycO with the Yop regulatory network is a more likely explanation. Since YopO and YopP proved to be dispensable for mediating the effect caused by SycO overproduction, we speculated on a role of the Yop regulatory proteins YscM1 and YscM2 in 


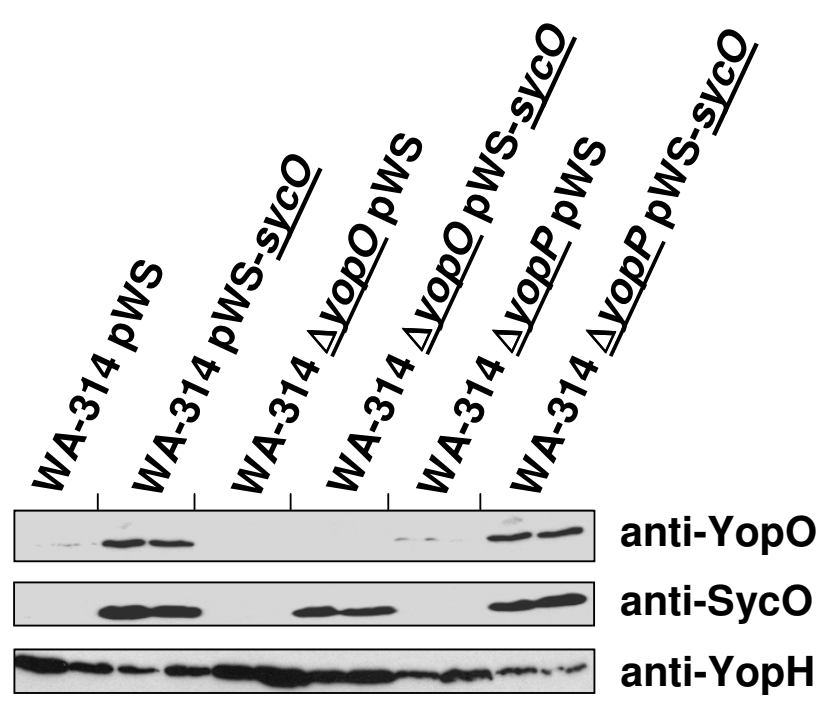

Figure 5

Differential effect of SycO overproduction on cytosolic Yop levels in the presence of calcium ions. Overproduction of SycO was analyzed in $Y$. enterocolitica parental strain WA-3 I4 and the isogenic deletion mutants $\triangle y o p O$ and $\triangle y o p P$ cultured at $37^{\circ} \mathrm{C}$ in the presence of $5 \mathrm{mM} \mathrm{CaCl}$. After $2 \mathrm{~h} \mathrm{I} \mathrm{mM}$ IPTG was added and cultivation was continued for another 2 h. Pellet fractions were analyzed by immunoblotting with sera raised aginst $\mathrm{YopO}$, SycO and $\mathrm{YopH}$.

this context. Clearly, neither YscM1 nor YscM2 was required to establish the SycO phenotype. Presumably, at least one further interaction partner of SycO has to be identified. This will help to understand whether SycO overexpression acts at the level of transcription or translation.

Nevertheless, we could demonstrate an interaction between YscM1 and SycO in vitro. In support of such an interaction, overproduction of SycO in the $y s c \mathrm{M} 1$ deletion strain influenced YopO and YopP secretion and intracellular accumulation of YopO. The YscM proteins are involved in the post-transcriptional regulation of Yop expression. In addition, they are T3S substrates themselves and chaperoned by $\mathrm{SycH}$, another actor in the Yop expression network $[25,36,37]$. Recently, binding of SycE to YscM1 and YscM2, and binding of SycD to YscM2 was demonstrated $[30,31]$. What could be the meaning of this YscM promiscuity? An unspecific interaction of both YscM1 and YscM2 with the acidic T3SS chaperones seems unlikely, given that the isoelectric point of YscM1 is 6.05 whereas the pI of YscM2 is 9.79. We propose that the YscM proteins function as an interface, integrating information on whether T3SS chaperones are loaded with Yops, and transducing these signals into repression or derepression of Yop translation.

\section{Conclusion}

This study supports the recently established view that SycO is a typical T3SS chaperone of class I that increases solubility and secretion efficiency of the effector YopO [20]. Beyond that, our results suggest that SycO is part of the T3SS regulatory network. This finding highlights the need for a complete T3SS interactome to understand the complex T3SS machinery.

\section{Methods \\ DNA manipulation}

Yersinia enterocolitica WA-314 [24]sycO was amplified by PCR using primers 5'-AAAAAACATATGATTAACTCAACCTTTACTGAGC-3' and 5'-ACTCGAGGTCGACTCAATAACCGATTGAGTAGATTGAGTAAG-3', thereby introducing NdeI and SalI restriction sites, respectively. The NdeI-SalI cleaved PCR product was introduced into expression vector pWS [18] resulting in plasmid pWSsycO. Y. enterocolitica WA-314 secB was cloned into pWS after generation of a PCR product using primers 5'-CATATGTCAGAACAAAACAACACCGAG-3' and 5'GTCGACTCAATGGTGATGGTGATGGTGGGCATCCT-

GACGTTGTTCAGC-3', and subcloning of the PCR product into pGEM-T (Promega). The resulting plasmid was termed pWS-secB. Construction of plasmid pWS-sycH was conducted in an analogous manner using primers 5'-CATATGCGCACTTACAGTTCATTAC-3' and 5'-GTCGACTTAAACCAGTAAATGAGATGATG-3'. Deletion of yscM1 and $y s c M 2$, respectively, in Y. enterocolitica WA-314 was introduced applying the phage lambda Red recombinase technique as previously established for $Y$. enterocolitica [35]. The kanamycin gene of plasmid pACYC177 (New England Biolabs) was amplified with 50-nucleotidehomology extensions corresponding to the flanks of the yscM1 coding region using primers 5'AATAAATAACTTAGAATATCGTAGAGATAATTATAGCGA CAGGAGACTCGTCACTGACACCCTCATCAGTG-3' and 5'-

ATCAACCTGGGGGTATTATCGCCTCAATATACAGTAAT ATATTATCGCGTCAAGTCAGCGTAATGCTC-3'. Analogously, $\quad$ primers 5'TGGTGGTTTAGTTTGTGTTTATTTTAAAATATACATGACA TATCGACGTTTCACTGACACCCTCATCAGTG-3' and 5'TGAAAAATTGAATTTTTAGTTTTAAGGTAACTTATCATTA AGTTGTGATACGTCAAGTTGTGATACGTCAAGT-

CAGCGTAATGCTC-3' were used in the case of yscM2. Construction of yopO and yopP deletion mutants was described previously [27].

\section{Culturing of yersiniae}

Induction of the T3SS of $Y$. enterocolitica WA-314 was accomplished essentially as described [38]. In brief, yersiniae overnight cultures grown at $27^{\circ} \mathrm{C}$ in brain heart infusion broth (BHI; Difco) were diluted 1:20 in BHI supplemented with $0.2 \mathrm{mM} \mathrm{CaCl}_{2}$ and cultured for $2 \mathrm{~h}$ at 

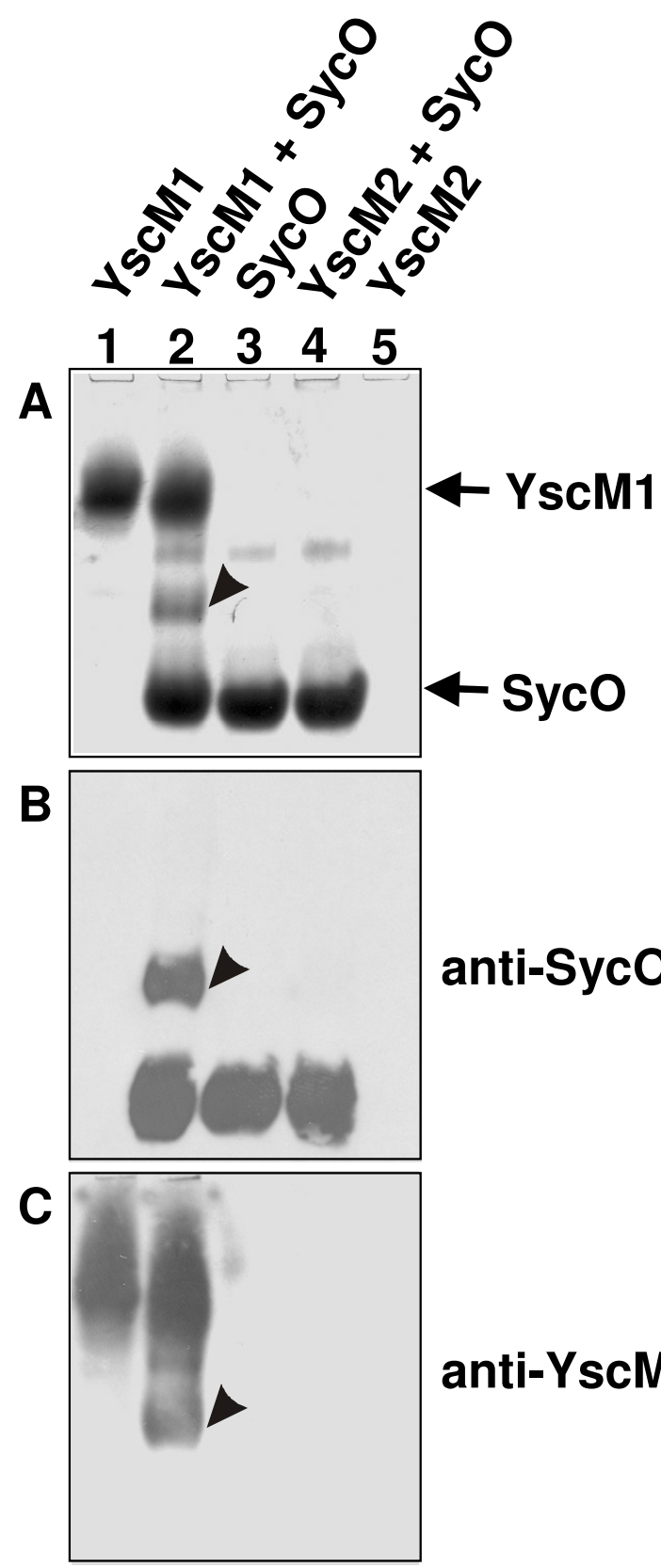

\section{anti-SycO}

\section{anti-YscM1}

\section{Figure 6}

Native gel electrophoresis reveals an interaction between SycO and YscMI. Recombinant proteins as indicated were loaded on Tris-buffered native gels. The gels were Coomassie-stained $(A)$ or alternatively electro-blotted and incubated with antisera raised against SycO (B), and YscMI (C), respectively. The amount of SycO $(20 \mu \mathrm{g})$ was approximately equimolar to that of YscMI (I5 $\mu \mathrm{g})$ and YscM2 (I $5 \mu \mathrm{g})$. SycO/ YscMI complex is indicated by arrowheads. YscM2 does not enter the native gel $(\mathrm{pH} 8)$ due to its high isoelectric point ( $\mathrm{pl}$ 9.79). $37^{\circ} \mathrm{C}$. Then, EGTA was added to a final concentration of $5 \mathrm{mM}$ and $\mathrm{MgCl}_{2}$ to a final concentration of $10 \mathrm{mM}$ for induction of Yop secretion. Alternatively, for repression of Yop secretion yersiniae were cultivated for $4 \mathrm{~h}$ at $37^{\circ} \mathrm{C}$ in BHI supplemented with $5 \mathrm{mM} \mathrm{CaCl}_{2}$. Overproduction of SycO and SecB was induced by addition of $1 \mathrm{mM}$ IPTG at the time of EGTA supplementation.

\section{Expression and purification of recombinant SycO}

The strategy for production of recombinant $\mathrm{SycO}$ was essentially as described for SycT [18]. Plasmid pWS-sycO was transformed into BL21 (DE3) pLysS (Stratagene). A $100 \mathrm{ml}$ overnight culture was grown in LB medium supplemented with $1 \%$ glucose at $37^{\circ} \mathrm{C}$ and diluted $1: 50$ in 5 liter LB medium. The culture was further grown in a BIOSTAT B fermenter (B.Braun Biotech International) at $37^{\circ} \mathrm{C}$ under conditions of constant aeration $(10 \mathrm{l} / \mathrm{min}), \mathrm{pH}$ (7.2) and stirring $(200 \mathrm{rpm})$. At an $\mathrm{OD}_{600}$ of 0.7 the temperature was decreased to $27^{\circ} \mathrm{C}$ and subsequently SycO expression was induced by adding $0.2 \mathrm{mM}$ IPTG. Bacteria were cultivated for another $18 \mathrm{~h}$, harvested by centrifugation and frozen at $-20^{\circ} \mathrm{C}$. The bacterial pellet from 1.25 liter of culture was resuspended in $80 \mathrm{ml}$ of Buffer A (50 $\mathrm{mM}$ Tris-HCl pH7.6, $100 \mathrm{mM} \mathrm{NaCl}, 2 \mathrm{mM}$ DTT) supplemented with $4 \mathrm{mM}$ PMSF and $250 \mathrm{U}$ Benzonase (Merck Biosciences). The sample was passed through a French press three times (1000 psi) and centrifuged at 20,000 g for $40 \mathrm{~min}$. The supernatant was adjusted to $20 \%$ $\left(\mathrm{NH}_{4}\right)_{2} \mathrm{SO}_{4}$ and gently stirred for $2 \mathrm{~h}$ at $4{ }^{\circ} \mathrm{C}$. The SycO precipitate was obtained by centrifugation $\left(20,000 \mathrm{~g}, 4^{\circ} \mathrm{C}\right)$, washed with precipitation buffer and resuspended in buffer A. The sample was then loaded on a MonoQ column (HiTrap Q FF, $5 \mathrm{ml}$; Amersham Biosciences) equilibrated with buffer A. The column was washed with $10 \mathrm{ml}$ of buffer A, then elution was conducted by applying a 100 $\mathrm{ml}$ linear gradient of $0-100 \%$ elution buffer ( $50 \mathrm{mM}$ Tris$\mathrm{HCl} \mathrm{pH} \mathrm{7.6,} 1 \mathrm{M} \mathrm{NaCl}, 1 \mathrm{mM}$ DTT). SycO eluted in the range of $280-370 \mathrm{mM} \mathrm{NaCl}$.

\section{Chemical cross-linking}

Cross-linking reagent DSP (dithiobis [succinimidylpropionate]) was purchased from Pierce. For cross-linking of SycO, $10 \mu \mathrm{l}$ reaction volume containing $1.3 \mathrm{mM}$ DSP and $3 \mu \mathrm{g} / \mu \mathrm{l}$ recombinant $\mathrm{SycO}$ in phosphate buffered saline (PBS) was incubated for $30 \mathrm{~min}$ at $22^{\circ} \mathrm{C}$. The reaction was stopped by adding $0.4 \mu \mathrm{l}$ of a $1 \mathrm{M}$ Tris- $\mathrm{HCl} \mathrm{pH} 7.5$ solution and was incubated for another $15 \mathrm{~min}$ at $22^{\circ} \mathrm{C} .5 \mu \mathrm{l}$ of SDS-PAGE loading buffer containing 0.5\% 2-mercaptoethanol were added and the sample was heated for 5 min at $95^{\circ} \mathrm{C}$ before loading.

\section{Native gel electrophoresis}

Tris-buffered continuous native gel electrophoresis was performed as previously described [31]. Gels were stained 


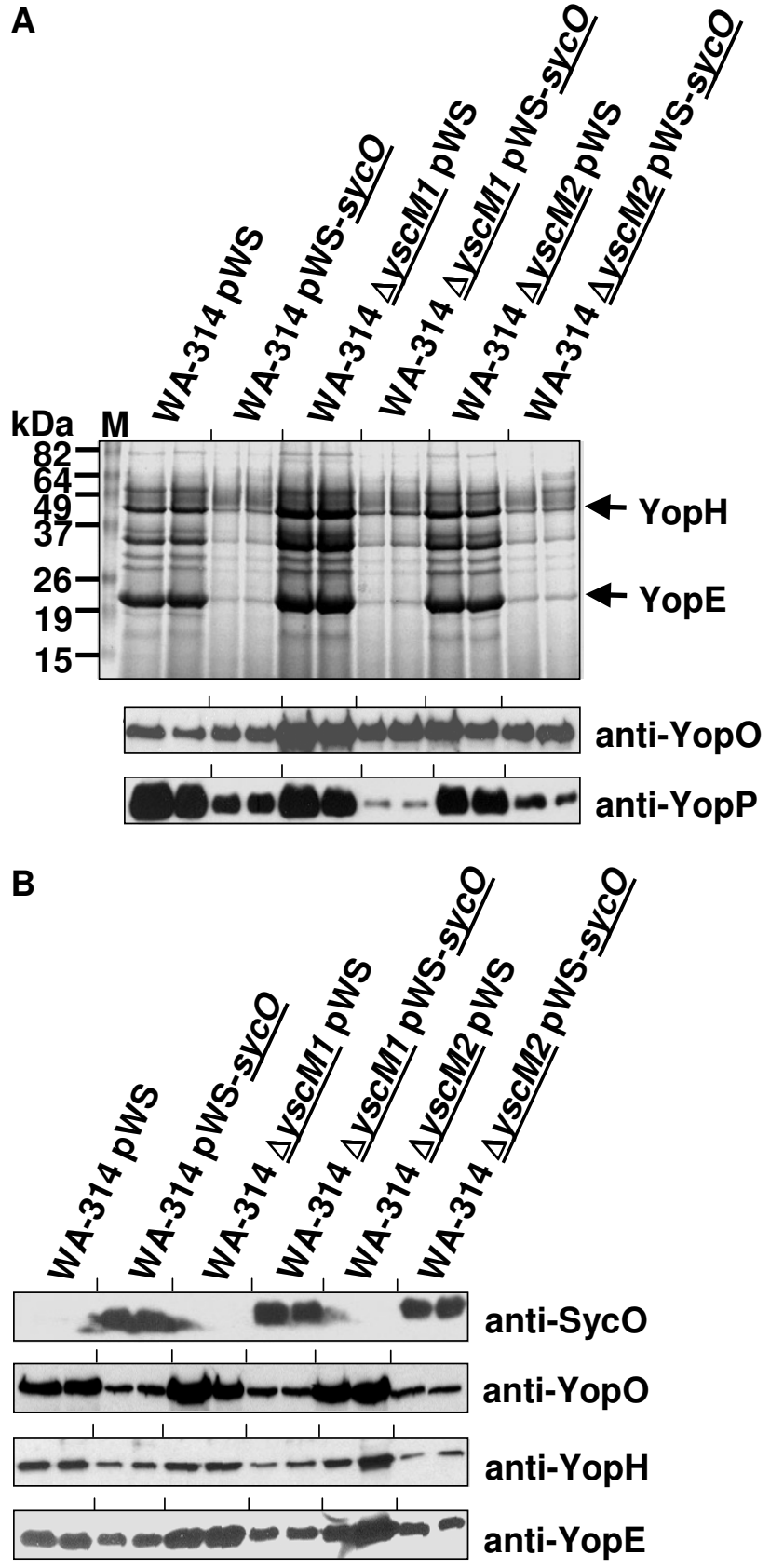

Figure 7

Repression of Yop secretion by SycO overproduction is neither mediated by YscMI nor by YscM2. Overproduction of SycO was analyzed in $Y$. enterocolitica parental strain WA-3 14 and the isogenic deletion mutants $\triangle y s c M I$ and $\triangle y s c M 2$ as described in legend to Fig. 2. Analysis of the culture supernatant is represented in (A) and the cell lysates are shown in (B).

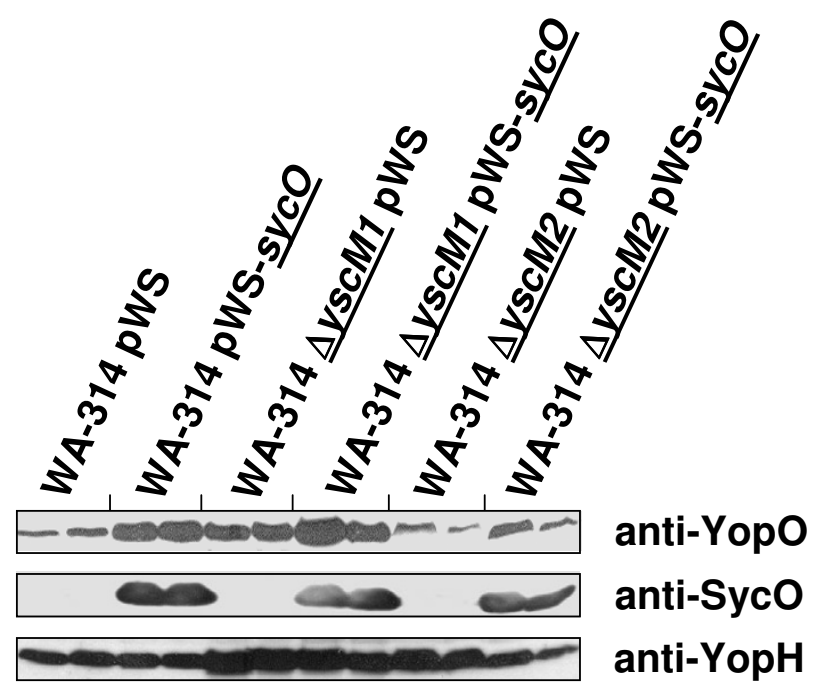

Figure 8

SycO overproduction in the mutant strains $\Delta y s c M I$ and $\triangle y s c M 2$ in the presence of calcium ions. Overproduction of SycO was analyzed in $Y$. enterocolitica parental strain WA-3I4 and the isogenic deletion mutants $\triangle y s c M I$ and $\triangle y s c M 2$ cultured at $37^{\circ} \mathrm{C}$ in the presence of $5 \mathrm{mM} \mathrm{CaCl}_{2}$. After $2 \mathrm{~h} \mathrm{I}$ mM IPTG was added and cultivation was continued for another $2 \mathrm{~h}$. Pellet fractions were analyzed by immunoblotting with sera raised aginst $\mathrm{YopO}, \mathrm{SycO}$ and $\mathrm{YopH}$.

with Coomassie Brilliant Blue or were alternatively electro-blotted.

\section{Analytical gel filtration chromatography}

Analytical gel filtration was performed with a SMART system (Amersham Biosciences) as described [33]. Recombinant SycO $(50 \mu \mathrm{g})$ and the monomeric $29 \mathrm{kDa}$ protein carbonic anhydrase $(50 \mu \mathrm{g})$ were mixed in a volume of 50 $\mu \mathrm{l}$ of PBS and loaded on a Superdex 75 PC 3.2/30 column (Amersham Biosciences) equilibrated with PBS. Fractions of $50 \mu \mathrm{l}$ were collected and analyzed by SDS-PAGE. Marker proteins for column calibration were purchased from Sigma (bovine serum albumine, $67 \mathrm{kDa}$; carbonic anhydrase, $29 \mathrm{kDa}$; cytochrome $c, 12.4 \mathrm{kDa}$ ).

\section{Authors' contributions}

$\mathrm{SD}, \mathrm{AS}, \mathrm{JH}$, and GW conceived of the study. SD carried out analytical gel filtrations, expression and secretion analyses, and edited the manuscript. AS performed native gel electrophoresis, chemical cross-linking experiments, and edited the manuscript. SR generated recombinant plasmids and performed expression and secretion analyses. KT generated deletion mutants and edited the manuscript. JH supervised the work, and edited the manuscript. GW coached the lab staff, and wrote the draft of the manu- 
script. All authors read and approved the final manuscript.

\section{Acknowledgements}

We thank Gabriele Liegl and Emilia Sieger for excellent technical assistance. This work was supported by the Deutsche Forschungsgemeinschaft (SFB 594 Teilprojekt B6).

\section{References}

I. Troisfontaines P, Cornelis GR: Type III secretion: more systems than you think. Physiology (Bethesda) 2005, 20:326-339.

2. Cornelis GR, Van Gijsegem F: Assembly and function of type III secretory systems. Annu Rev Microbiol 2000, 54:735-774.

3. Stebbins CE, Galan JE: Maintenance of an unfolded polypeptide by a cognate chaperone in bacterial type III secretion. Nature 200I, 4|4:77-8I.

4. Birtalan SC, Phillips RM, Ghosh P: Three-dimensional secretion signals in chaperone-effector complexes of bacterial pathogens. Mol Cell 2002, 9:97I-980.

5. Schubot FD, Jackson MW, Penrose KJ, Cherry S, Tropea JE, Plano GV, Waugh DS: Three-dimensional structure of a macromolecular assembly that regulates type III secretion in Yersinia pestis . J Mol Biol 2005, 346: I I 47-II6I.

6. Luo Y, Bertero MG, Frey EA, Pfuetzner RA, Wenk MR, Creagh L, Marcus SL, Lim D, Sicheri F, Kay C, Haynes C, Finlay BB, Strynadka NC: Structural and biochemical characterization of the type III secretion chaperones CesT and SigE. Nat Struct Biol 200I, 8: $1031-1036$.

7. Neumayer W, Groll M, Lehmann V, Antoneka U, Kähler S, Heesemann J, Wilharm G: Yersinia enterocolitica type III secretion chaperone SycH. Recombinant expression, purification, characterisation, and crystallisation. Protein Expr Purif 2004, 35:237-247.

8. Wilharm G, Lehmann V, Neumayer W, Trèek J, Heesemann J: Yersinia enterocolitica type III secretion: evidence for the ability to transport proteins that are folded prior to secretion. $B M C$ Microbiol 2004, 4:27.

9. Gauthier A, Finlay BB: Translocated intimin receptor and its chaperone interact with ATPase of the type III secretion apparatus of enteropathogenic Escherichia coli . J Bacteriol 2003, 185:6747-6755.

10. Akeda $Y$, Galan JE: Chaperone release and unfolding of substrates in type III secretion. Nature 2005, 437:9II-9/5.

II. Cornelis GR: How Yops find their way out of Yersinia . Mol Microbiol 2003, 50:1091-1094.

12. Ramamurthi KS, Schneewind O: Substrate recognition by the Yersinia type III protein secretion machinery. Mol Microbiol 2003, 50:1095-1102.

13. Birtalan S, Ghosh P: Structure of the Yersinia type III secretory system chaperone SycE. Nat Struct Biol 200I, 8:974-978.

14. Evdokimov AG, Tropea JE, Routzahn KM, Waugh DS: Threedimensional structure of the type III secretion chaperone SycE from Yersinia pestis. Acta Crystallogr D Biol Crystallogr 2002, 58:398-406.

15. Trame CB, McKay DB: Structure of the Yersinia enterocolitica molecular-chaperone protein SycE. Acta Crystallogr D Biol Crystallogr 2003, 59:389-392

16. Phan J, Tropea JE, Waugh DS: Structure of the Yersinia pestis type III secretion chaperone SycH in complex with a stable fragment of YscM2. Acta Crystallogr D Biol Crystallogr 2004, 60:1591-1599.

17. Buttner CR, Cornelis GR, Heinz DW, Niemann HH: Crystal structure of Yersinia enterocolitica type III secretion chaperone SycT. Protein Sci 2005, 8:1993-2002.

18. Locher M, Lehnert B, Krauss K, Heesemann J, Groll M, Wilharm G: Crystal structure of the Yersinia enterocolitica type III secretion chaperone SycT. J Biol Chem 2005, 280:3 I I49-3 II 55.

19. Ghosh P: Process of protein transport by the type III secretion system. Microbiol Mol Biol Rev 2004, 68:771-795.

20. Letzelter M, Sorg I, Mota LJ, Meyer S, Stalder J, Feldman M, Kuhn M, Callebaut I, Cornelis GR: The discovery of SycO highlights a new function for type III secretion effector chaperones. $E M B O$ J 2006, 25:3223-3233.
21. Galyov EE, Hakansson S, Wolf-Watz $\mathrm{H}$ : Characterization of the operon encoding the YpkA Ser/Thr protein kinase and the YopJ protein of Yersinia pseudotuberculosis . J Bacteriol 1994, 176:4543-4548.

22. Perry RD, Straley SC, Fetherston JD, Rose DJ, Gregor J, Blattner FR: DNA sequencing and analysis of the low-Ca2+-response plasmid pCDI of Yersinia pestis KIM5. Infect Immun 1998, 66:4611-4623.

23. Trülzsch K, Roggenkamp A, Aepfelbacher M, Wilharm G, Ruckdeschel K, Heesemann J: Analysis of chaperone-dependent Yop secretion/translocation and effector function using a minivirulence plasmid of Yersinia enterocolitica . Int J Med Microbiol 2003, 293: 167-177.

24. Heesemann J, Laufs R: Construction of a mobilizable Yersinia enterocolitica virulence plasmid. J Bacteriol 1983, 155:76|-767.

25. Cambronne ED, Cheng LW, Schneewind O: LcrQ/YscMI, regulators of the Yersinia yop virulon, are injected into host cells by a chaperone-dependent mechanism. Mol Microbiol 2000, 37:263-273.

26. Wulff-Strobel CR, Williams AW, Straley SC: LcrQ and SycH function together at the Ysc type III secretion system in Yersinia pestis to impose a hierarchy of secretion. Mol Microbiol 2002, 43:4II-423.

27. Trülzsch K, Sporleder T, Igwe El, Rüssmann H, Heesemann J: Contribution of the major secreted Yops of Yersinia enterocolitica O:8 to pathogenicity in the mouse infection model. Infect Immun 2004, 72:5227-5234.

28. Bergman T, Hakansson S, Forsberg A, Norlander L, Macellaro A, Backman A, Bolin I, Wolf-Watz H: Analysis of the $\mathbf{V}$ antigen IcrGVH-yopBD operon of Yersinia pseudotuberculosis : evidence for a regulatory role of LcrH and LcrV. J Bacteriol I99I, 173:1607-1616

29. Forsberg A, Wolf-Watz $\mathrm{H}$ : Genetic analysis of the yopE region of Yersinia spp.: identification of a novel conserved locus, yerA, regulating yopE expression. J Bacteriol 1990, 172:1547-1555.

30. Swietnicki W, O'Brien S, Holman K, Cherry S, Brueggemann E, Tropea JE, Hines HB, Waugh DS, Ulrich RG: Novel protein-protein interactions of the Yersinia pestis type III secretion system elucidated with a matrix analysis by surface plasmon resonance and mass spectrometry. J Biol Chem 2004, 279:38693-38700.

31. Schmid A, Dittmann S, Grimminger V, Walter S, Heesemann J, Wilharm G: Yersinia enterocolitica type III secretion chaperone SycD: recombinant expression, purification and characterization of a homodimer. Protein Expr Purif 2006, 49: 176-182.

32. Stainier I, Iriarte M, Cornelis GR: YscMI and YscM2, two Yersinia enterocolitica proteins causing downregulation of yop transcription. Mol Microbiol 1997, 26:833-843.

33. Wilharm G, Neumayer W, Heesemann J: Recombinant Yersinia enterocolitica YscMI and YscM2: homodimer formation and susceptibility to thrombin cleavage. Protein Expr Purif 2003, 31:167-172.

34. Datsenko KA, Wanner BL: One-step inactivation of chromosomal genes in Escherichia coli K-I 2 using PCR products. Proc Natl Acad Sci USA 2000, 97:6640-6645.

35. Wilharm G, Lehmann V, Krauss K, Lehnert B, Richter S, Ruckdeschel K, Heesemann J, Trülzsch K: Yersinia enterocolitica type III secretion depends on the proton motive force but not on the flagellar motor components MotA and MotB. Infect Immun 2004, 72:4004-4009.

36. Pettersson J, Nordfelth R, Dubinina E, Bergman T, Gustafsson M, Magnusson KE, Wolf-Watz H: Modulation of virulence factor expression by pathogen target cell contact. Science 1996, 273:|23|-|233

37. Cambronne ED, Schneewind O: Yersinia enterocolitica type III secretion: yscMI and yscM2 regulate yop gene expression by a posttranscriptional mechanism that targets the 5 ' untranslated region of yop mRNA. J Bacteriol 2002, I 84:5880-5893.

38. Trček J, Wilharm G, Jacobi CA, Heesemann J: Yersinia enterocolitica YopQ: strain-dependent cytosolic accumulation and post-translational secretion. Microbiology 2002, I48: I457-I 465. 